\title{
Herbal Products Use During Pregnancy in North of Iran
}

\author{
Gelareh Biazar $^{1}$, Bahram Naderi Nabi² ${ }^{2}$ Abbas Sedighinejad', Anoush Dehnadi Moghadam ${ }^{3}$, Farnoush \\ Farzi $^{*}{ }^{*}$, Zahra Atrkarroushan ${ }^{4}$, Farimah Mirblook ${ }^{4}$, Ladan Mirmansouri ${ }^{5}$
}

\begin{abstract}
Objectives: The use of herbs which has been increasing worldwide, requires special considerations particularly in pregnant woman. The present study was conducted at Alzahra Teaching hospital (from March 2016 to August 2017) to explore the pattern of herbal use during pregnancy in Guilan province (North of Iran).

Materials and Methods: Data were obtained from 836 eligible women, who were interviewed at postnatal ward, and were analyzed. Results: The results of the study showed that $19.6 \%$ of the women consumed herbs during pregnancy. The most commonly used herbs included mint, flixweld, and cinnamon; in addition, the most prevalent indications resulted from these herbs consumption were gastrointestinal complications and cold. The rate of herbal consumption in pregnancy was related to education $(P=0.001)$ and resistance $(P=0.008)$; however, no relation was found regarding age $(P=0.203)$, employment $(P=0.255)$, or gravity $(P=0.935)$.

Conclusions: Although the findings of this study were preferable to those of some other studies, due to the importance of the issue, it is vital that health care providers to be open to question the herbal use during pregnancy.

Keywords: Herbal products, Pregnancy, North of Iran
\end{abstract}

\section{Introduction}

Herbal products are frequently used for some pregnancy related complaints such as cough, cold, and gastrointestinal problems. However, their safety and effectiveness are still being studied. In fact, among so many herbs, only ginger has been approved by the Food and Drug Administration (FDA) (1). Besides, unlike chemical drugs, the exact safe and effective dosage and interval of herbal products are not determined, yet. Due to their active components, severe interactions between herbs and medical drugs should also be considered (2). Therefore, although some experimental and clinical studies support the herbal medicine as a cost effective alternative with beneficial effects such as antioxidant properties, it should be avoided during pregnancy(3-6).

Healthy intake of the herbal medicine is of great importance during pregnancy since it supports an appropriate fetus growth and safety. However, these products, due to several reasons, are taken by the pregnant woman without a physician recommendation. They consider herbs less harmful compared with chemical treatments. Cost effectiveness and accessibility might be the other reasons for the consumption of such herbs. According to what has been found, as high as sixty percent of the women use herbal therapies during pregnancy (7). However, the true incidence of herbal use during pregnancy is not known in Iran particularly in Guilan province owing to lack of enough investigation in this regard. Although many studies have been conducted in this field, they have all discovered herbal supplementation among pregnant women in other countries. To the best knowledge of the researchers, no similar study has been carried out in North of Iran. In fact, there is no data about the pattern of herbal use during pregnancy in this area. Accordingly, considering the importance of the issue under question and the difference in vegetation cover of various regions of Iran, the present study was designed. Obviously, the next step would be changing pregnant women' attitudes and improving their knowledge in this respect. In the current study, pattern of herbal use by pregnant women in Guilan province was explored. As Alzahra hospital is a referral center with patients from private and public hospitals, the results would be valuable and practical.

\section{Materials and Methods}

This descriptive cross-sectional study took place in a university hospital affiliated to Guilan University of Medical Sciences (GUMS). The hospital is the only referral center in the area with almost 4000 deliveries annually.

\section{Sample Size}

With an approximation of 4000 births per year at Alzahra hospital, single population proportion formula was used 
with the assumption of $95 \% \mathrm{CI}$ and $5 \%$ margin of error; no respondents were presumed of $5 \%$ and a final sample size of 836 was determined.

A total of 836 eligible women were interviewed within 2 days after childbirth (from March 2016 to August 2017).

Inclusion Criteria: Mothers with none-malformed babies from Guilan province.

Exclusion Criteria: A mother or a baby admitted to intensive care unit (ICU) or a mother who refused to give an informed consent.

At first, the aim of the study was explained to all the individuals. Then, the exact definition of herbal product, that is, any type of drugs with any root of administration oral or dermal, was provided to them, and all their questions were precisely answered. A standardized questionnaire was filled by a trained nurse through a face to face interview. The validity and reliability of the questionnaire were confirmed by ten members of Anesthesiology Department.

\section{Data Analysis}

To statistically analyze the data, SPSS (Statistical Package for the Social Sciences) software, version 16 was used. Appropriate descriptive statistics were applied as well. In addition, Fisher exact tests were used for categorical variables.

\section{Results}

Totally, data from 836 women who were interviewed within two days after childbirth were analyzed. Nearly, 19.6\% of them reported that they had taken herbal products during their pregnancy. The socio-demographic and pregnancy related characteristics of the respondents are summarized in Table 1. Besides, the most commonly used herbal medicines and indications during pregnancy are presented in Table 2. The amount of herbal consumption in pregnancy was related to education $(P=0.001)$ and resistance $(P=0.008)$; however no relation was observed with regard to age $(P=0.203)$, employment $(P=0.255)$, or gravity $(P=0.935)$.

Table 1. Socio-Demographic Characteristics and Factors Associated With Herbal Medicine Use Among Respondents

\begin{tabular}{llc}
\hline Variables & Statue & No. (\%) \\
\hline Age groups (y) & $\leq 20$ & $219(25.3)$ \\
& $20-30$ & $467(54.1)$ \\
& $\geq 30$ & $178(20.6)$ \\
Education & Illiterate & $13(1.5)$ \\
& Primary & $280(32.4)$ \\
& Tertiary & $489(56.8)$ \\
& Universal & $82(9.5)$ \\
Employment status & Employed & $33(3.8)$ \\
& Unemployed & $831(96.2)$ \\
Residence & Urban & $625(72.3)$ \\
& Rural & $239(27.7)$ \\
\hline
\end{tabular}

\section{Discussion}

The adverse effects of some herbs such as daily almond oil and chamomile have been confirmed in pregnant women; these herbs were found to be associated with preterm labor and miscarriage (8). In addition to their potential risks for the fetus and the mother, when due to the obstetric indications cesarean section is planned, certain herbal products might have serious interactions with regional or general anesthesia drugs (9). A wash out time is well defined for each herb. However, if the type of the consumed herb is unknown, it is recommended to stop it at least two weeks before the anesthesia (10).

In spite of the risk for teratogenicity of these products during pregnancy, our knowledge and informing strategies have not been improved constant with the rapidly rise in their consumption rate. It is reported that only $10.2 \%$ of the pregnant women believed that, within their visits, they had to inform their medical team about herbal use. This inappropriate communication led to severe adverse effects on the mother and her fetus (11). In fact, one of the important roles of health care providers should be asking the pregnant women about the herbal use. The findings of this study revealed that $19.6 \%$ of the cases consumed herbs during their pregnancy. Comparing these results with the findings of the other similar epidemiological studies, it was found that pregnant women in Guilan province were less prone to herbal intake. In the present study, a positive relation was found between herbal treatments and residency and education, but, this consistency was not observed regarding age and gravity variables. An increased rate of herbal use was found in urban residence and higher educated women. In spite of the fact that the effectiveness of herbal use in pregnancy is not an acceptable attitude, focusing on the indications of each herb use is partly based on the knowledge obtained in this regard. For instance, having knowledge about the therapeutic effects of green tea for weight loss; cinnamon for blood sugar control; saffron for abortion; ginger for nausea and vomiting; and cowslip for relaxation. Therefore, the findings of the current study were constant because in urban population, higher education and consequently more knowledge were expected on the part of the pregnant woman.

Several studies indicated that woman with white ethnicity, high level of education and outcome and also those who are middle-aged are more subject to herbal use. Marwa et al reported that the prevalence of herbal use among pregnant women was high in Tanzania. About $33.1 \%$ of them used herbs which was a threat to the safety of the developing fetus and as a result, there was a need for interventions. Headache, malaria, and morning sickness were the most common causes that necessitated selfmedication among pregnant woman (12). In addition, John and Shantakumari, in a review article indicated that the rate of herbal supplementation in different forms varied between $22.3 \%-82.3 \%$ in middle east with a more tendency among pregnant women (13). Facchinetti et al, 
Table 2. The Most Commonly Used Herbal Medicines and Indications During Pregnancy

\begin{tabular}{|c|c|c|c|c|}
\hline Persian Name of the Herbs & English Name of the Herbs & No. & $\%$ & Most Common Reasons Reported for Indication \\
\hline Darchin & Cinnamon & 34 & 15.4 & Reducing Blood Sugar/For Male Gender \\
\hline Nana & Mint & 33 & 14.9 & GI Problems/UTI \\
\hline Khakshir & Flixweld & 29 & 13.1 & Common Cold \\
\hline Taranjabeen & Manna & 21 & 9.4 & Preventing Neonatal Jaundice \\
\hline Kasni & Chicory & 21 & 9.4 & Relaxation \\
\hline Golgavzaban & Cowslip & 14 & 6.3 & Relaxation/Common Cold \\
\hline Zanjebeel & Ginger & 11 & 5 & Nausea \&Vomiting \\
\hline Zireh & Cumin & 10 & 4.5 & GI Problems \\
\hline Avishan & Thyme & 9 & 4 & GI Problems \\
\hline Chayesabz & Green tea & 7 & 3.2 & Weight Loss \\
\hline Kondor & Olibanum & 6 & 2.7 & Intelligent Baby \\
\hline Babooneh & Chamomile & 6 & 2.7 & Common Cold \\
\hline Pooneh & Oregano & 6 & 2.7 & Nausea \&Vomiting \\
\hline Onab & Jujube Tree & 4 & 1.8 & Common Cold \\
\hline Tokhm-e- sharbati & Sweet Basil & 4 & 1.4 & Common Cold \\
\hline Razianeh & Fennel & 4 & 1.8 & Muscle Pain \\
\hline $\mathrm{Hel}$ & Cardamom & 3 & 1.4 & Beautiful Baby \\
\hline Zaferan & Saffron & 3 & 1.4 & Induced Abortion \\
\hline
\end{tabular}

in a cohort retrospective study in Italy, also reported that $42 \%$ of the women were likely to be the users of herbs during their pregnancy. Almond oil and chamomile which were used for stretch marks and anxiety were the most used herbs (8). Similarly, Sereshty et al, showed that $51.9 \%$ of pregnant women in Shahr-e Kord used the herbs. Unfortunately, they reported that $91.7 \%$ of the referred women, believed that the use of herbal medicines was safe during pregnancy (9).

There are some possible explanations for the different results obtained by similar studies. The prevalence of herbal medicine use during pregnancy depends on culture, believes, and geographic features (11, 14). Moreover, definitions of herbal products are not the same. Sometimes all forms of natural remedies and any supplement containing even one vitamin are considered as herbal or might be so restricted. In their study in Ethiopia, Mekuria et al, questioned a wide range of herb products such as any form of garlic consumption and reported that nearly $48.6 \%$ of their cases had herbal use (11). Refuerzo et al, in a prospective observational study conducted at a single tertiary-care hospital, evaluated women in postpartum ward and reported that $96.9 \%$ of them used at least one medication during pregnancy. This result was achieved administering a questionnaire which contained 120 items including the herbs, supplementations, and other alternative therapies. However, after excluding all the items expect for the herbs, this percent diminished to $4.1 \%$ (15). In addition, the results were mainly affected by the study inclusion criteria. Tabatabaee in Fars (14) and Kissal et al in Turkey (2) enrolled only mothers of healthy babies. Herbal supplementation rate among pregnant women in their studies was found to be $30.8 \%$ and $47.3 \%$, respectively. However, in the studies conducted in Ethiopia (11) and Shahrekord (7), mothers with malformed babies or those who were admitted to ICU were not excluded from the study and the percentage of herbal use in their studies was $69.8 \%$ and $51.9 \%$, respectively. Due to the probability of amnesia, the time of interview with the newly-delivered mothers was also important. In another study by Mothupi, in Kenya, the exposure rate was identified through an interview within 9 months of child birth (16); however, in Facchinetti and colleagues' study this time was restricted to three days (8). They reported herbal use of $12 \%$ and $47.2 \%$, respectively.

\section{Limitations}

In every human attempt, no doubt, there exist some limitations and problems which need to be acknowledged. The reporting in this study may not be complete because some infrequently consumed herbs data could be affected by the amnesia. In this study, only mothers of healthy infants were enrolled. Surely, the results would be different and more reliable if all the women with dead or malformed babies had not been excluded. Therefore, the results of the study may not be generalized. In addition, this study was conducted in a teaching and public single center. Other cases from private hospitals might be different with regard to culture, economy, and education.

\section{Strengths of the Study}

This study had some strengths which also need to be acknowledged. The participants of this study were questioned through a face to face interview and within two days postpartum. Therefore, the findings might be more accurate compared to the results of the studies 
which evaluated their cases by sending questionnaires and also interviewing after a long time following the delivery.

Suggestions for Further Research

Due to the wide range of differences within the areas of our country, namely, north, south, east, and west in terms of plants coverage, believes, and cultures, more studies are required in order to particularly delve into the issue. Surely, different results will be achieved in doing so. Moreover, as previously mentioned, the study was carried out in a teaching and public single center. Other pregnant women from private hospitals might be different culturally, economically, and academically. As a result, similar studies focusing on other cases from a variety of contexts are subject to further investigation.

\section{Conclusions}

Despite the lower rate of herbal use as compared to some other studies, considering the importance of saving two lives, not all the herbal supplementations should be ignored. All pregnant women should be questioned within their periodical visits and attempts should be made to improve their attitude and knowledge in this regard. Obviously, even the lowest risks can overcome the benefits.

\section{Conflict of Interests}

Authors declare that they have no conflict of interests.

\section{Ethical Issues}

The study protocol was approved by the Research Ethics Committee of Guilan University of Medical Sciences (Ethics No. 1396.83).

\section{Financial Support}

This work was supported by Anesthesiology Research Center of Guilan University of Medical Sciences.

\section{Acknowledgments}

The researchers thank Mrs. Fatemeh Rashidi Mojdehi for her kind assistance in data collection process.

\section{References}

1. Sabet B, Salkhordeh H, Hedayati Khalil M, Mahboobi M, Babajani S. A Review of the Ginger Products Use in Reducing Pregnancy Nausea and Vomiting. Paramedical Sciences and Military Health. 2017;11(4):50-56.

2. Kissal A, Cevik Guner U, Batkın Erturk D. Use of herbal product among pregnant women in Turkey. Complement Ther Med. 2017;30:54-60. doi:10.1016/j.ctim.2016.11.001

3. Ouladsahebmadarek E, Seyed Giasi G, Khaki A, et al.
The Effect of Compound Herbal Remedy Used in Male Infertility on Spermatogenesis and Pregnancy Rate. Int J Women's Health Reproduction Sci. 2016;4(4):185-188. doi:10.15296/ijwhr.2016.41

4. Fathiazad F, khaki A, Nouri M, Khaki AA. Effect of Cinnamon Zeylanicum on serum Testosterone and antioxidants levels in Rats. Int J Women's Health Reproduction Sci. 2013;1(1):29-35.

5. Soltani M, Moghimian M, Abtahi H, Shokoohi M. The Protective Effect of Matricaria chamomilla Extract on Histological Damage and Oxidative Stress Induced by Torsion/Detorsion in Adult Rat Ovary. Int J Women's Health Reproduction Sci. 2017;5(3):187-192. doi:10.15296/ ijwhr.2017.34

6. Khaki A, Ainehchi N. Herbal Medicine and Sexual Behavior in Diabetic Patients. Int J Women's Health Reproduction Sci. 2017;5(1):1-2. doi:10.15296/ijwhr.2017.01

7. Jaafari M, Fotoohi A, Razavi N, Abdian Asl A. Herbal medicine in pregnancy. Adv Herb Med. 2016;2(1):54-66.

8. Facchinetti F, Pedrielli G, Benoni G, et al. Herbal supplements in pregnancy: unexpected results from a multicentre study. Hum Reprod. 2012;27(11):3161-3167. doi:10.1093/humrep/des303

9. Sereshty M, Azari P, Rafiean M, Kheiri S. Use of herbal medicines by pregnant women in Shahr-e-Kord. J Reprod Infertil. 2006;7(2):125-132.

10. Wang C, Yuan C, Moss J. Anesthetic implications of complementary and alternative medicathons. In: Miller's Anesthesia. Canada: Anne Altepeter; 2015:1227-29.

11. Mekuria AB, Erku DA, Gebresillassie BM, Birru EM, Tizazu B, Ahmedin A. Prevalence and associated factors of herbal medicine use among pregnant women on antenatal care follow-up at University of Gondar referral and teaching hospital, Ethiopia: a cross-sectional study. BMC Complement Altern Med. 2017;17(1):86. doi:10.1186/ s12906-017-1608-4

12. Marwa KJ, Njalika A, Ruganuza D, Katabalo D, Kamugisha E. Self-medication among pregnant women attending antenatal clinic at Makongoro health centre in Mwanza, Tanzania: a challenge to health systems. BMC Pregnancy Childbirth. 2018;18(1):16. doi:10.1186/s12884-017-1642-8

13. John LJ, Shantakumari N. Herbal Medicines Use During Pregnancy: A Review from the Middle East. Oman Med J. 2015;30(4):229-236. doi:10.5001/omj.2015.48

14. Tabatabaee M. Use of Herbal Medicine among Pregnant Women Referring to Valiasr Hospital in Kazeroon, Fars, South of Iran. Journal of Medicinal Plants. 2011;1(37):96108.

15. Refuerzo JS, Blackwell SC, Sokol RJ, et al. Use of over-thecounter medications and herbal remedies in pregnancy. Am J Perinatol. 2005;22(6):321-324. doi:10.1055/s-2005-873235

16. Mothupi MC. Use of herbal medicine during pregnancy among women with access to public healthcare in Nairobi, Kenya: a cross-sectional survey. BMC Complement Altern Med. 2014;14:432. doi:10.1186/1472-6882-14-432

(C) 2018 The Author (s); This is an open-access article distributed under the terms of the Creative Commons Attribution License (http://creativecommons.org/licenses/by/4.0), which permits unrestricted use, distribution, and reproduction in any medium, provided the original work is properly cited. 